\title{
359 RESULTS FROM A PHASE II STUDY OF EFTILAGIMOD ALPHA (SOLUBLE LAG-3 PROTEIN) AND PEMBROLIZUMAB IN PATIENTS WITH PD-L1 UNSELECTED METASTATIC 2ND LINE HEAD AND NECK SQUAMOUS CELL CARCINOMA (HNSCC)
}

${ }^{1}$ Antonio López Pousa*, ${ }^{2}$ Enriqueta Felip, ${ }^{3}$ Martin Forster, ${ }^{4}$ Bernard Doger, ${ }^{5}$ Patricia Roxburgh, ${ }^{6}$ Pawan Bajaj, ${ }^{7}$ Julio Peguero, ${ }^{8}$ Enric Carcereny, ${ }^{9}$ Matthew Krebs, ${ }^{10}$ Christian Mueller, ${ }^{11}$ Frederic Triebel. ${ }^{1}$ Hospital Sant Pau, Barcelona, Spain; ${ }^{2}$ Vall d'Hebron University Hospital, Barcelona, Spain; ${ }^{3}$ UCL Cancer Institute/NHS Foundation, London, UK; ${ }^{4}$ START Madrid- Fundación Jiménez Diaz, Madrid, Spain; ${ }^{5}$ Beatson West of Scotland Cancer Center, Glasgow, UK; ${ }^{6}$ Tasman Oncology, Queensland, Australia; ${ }^{7}$ Oncology Consultants, P. A., Houston, TX, USA; ${ }^{8}$ Catalan Institute of Oncology Badalona, Badalona, Spain; ${ }^{9}$ Christie NHS Foundation Trust, Manchester, UK; ${ }^{10}$ Clinical Development, Immutep GmbH, Berlin, Germany; ${ }^{11}$ Research and Development, Immutep S.A.S., Chatenay Malabry, France

Background Eftilagimod alpha (efti) is a soluble LAG-3 protein targeting a subset of MHC class II, thus mediating antigen presenting cell (APC) and CD8 T-cell activation. Such stimulation of the dendritic cell network and resulting $\mathrm{T}$ cell recruitment by efti may lead to stronger anti-tumor responses than observed with pembrolizumab alone. We hereby report results of the 2nd line metastatic head and neck squamous cell carcinoma (HNSCC) cohort (part C) of the TACTI-002 phase II trial (NCT03625323).

Methods Eligible patients (pts) with HNSCC, unselected for PD-L1 expression with disease progression on or after 1st line platinum-based therapy, received $30 \mathrm{mg}$ subcutaneous efti Q2W for 24 weeks and $200 \mathrm{mg}$ pembrolizumab Q3W for up to 2 years or until disease progression. The study used a Simon's 2-stage design with objective response rate (ORR) as the primary endpoint (EP). Secondary EPs included tolerability, progression free survival (PFS), overall survival (OS), pharmacokinetics, pharmacodynamics, and immunogenicity. Tumor response was assessed Q9W. PD-L1 was assessed centrally (22C3 clone). The study was approved by ethic committees and institutional review boards.

Results Between Mar 2019 and Jan 2021, 39 pts were enrolled (cut-off Apr 2021). The median age was 62 yrs (range 37-84) with 90\% male pts. ECOG was 0 and 1 in $33 \%$ and $67 \%$, respectively. Primary tumor location at diagnosis was oropharynx (36\%), oral cavity (31\%), hypopharynx $(18 \%)$ and larynx $(15 \%)$ with all PD-L1 subgroups $(\mathrm{CPS}<1$, $\geq 1$ to $\leq 19 ; \geq 20$ ) being represented. All pts were pre-treated with platinum-based chemotherapy. Thirty-seven (37) pts were evaluated for response with ORR (iRECIST) of 30\% (95\% CI 16-47\%) with 5 (14\%) CRs; 6 (16\%) PRs; 3 (8\%) SDs; 17 $(46 \%)$ PDs and $6(16 \%)$ pts not evaluable. Median PFS was 2.1 months and $30+\%$ were progression-free at 6 months. One patient $(3 \%)$ discontinued due to pembro-related adverse event. The most common $(>10 \%)$ treatment emergent adverse events were hypothyroidism (21\%), cough (18\%), asthenia $(15 \%)$, fatigue (13\%), anemia (13\%), diarrhea (13\%) and weight decreased (13\%).

Conclusions Efti in combination with pembrolizumab is safe, showing encouraging antitumor activity in platinum pretreated, 2nd line HNSCC patients. For further investigation of this combination, a 1st line HNSCC trial (NCT04811027) has been initiated.

Trial Registration NCT03625323

Ethics Approval The study was approved by relevant ethic committees and institutional review boards

http://dx.doi.org/10.1136/jitc-2021-SITC2021.359 